\title{
Opções Contemporâneas de Rastreio da Cardiotoxicidade Relacionada a Tratamentos Oncológicos
}

https://doi.org/10.32635/2176-9745.RBC.2019v65n3.388

\author{
Contemporaneous Screening Options of Cardiotoxicity Related to Oncological Treatment \\ Opciones Contemporáneas para el Rastreo de Cardiotoxicidad Relacionada con el Tratamiento del Cancer
}

Bruno de Henrique Rala de Paula; Lívia Liberata Barbosa Bandeira²; Alex Pereira Ramos³; Barbara Luiza Meireles Pinheiro4; Ramon Magro Ferreira5; Indiara Iris de Oliveira Araújo ${ }^{6}$; Pedro Crocamo Ventilari da Costa7; Susanne Crocamo ${ }^{8}$

\section{INTRODUÇÃO}

Os recentes avanços das terapias oncológicas permitiram a individualização do tratamento e estáo associados a ganho de sobrevida ${ }^{1}$. Em consonância, houve um aumento na incidência de efeitos colaterais ligados à toxicidade cardíaca bem como no surgimento de novas etiologias da injúria cardiovascular ${ }^{2}$.

O rastreamento da cardiotoxicidade, extrapolado dos regimes utilizados nos estudos pivotais, é realizado classicamente por meio da mensuração de risco pré-tratamento por exame físico precedido de anamnese associado a eletrocardiograma e da mensuração da fração de ejeção do ventrículo esquerdo (FEVE) pelo ecocardiograma ou pelo Multigated Acquisition Scan (MUGA Scan) ${ }^{3}$.

Entretanto, estudos recentes reportam maior sensibilidade de novas opçóes em potencial de rastreio, como a dosagem da troponina ultrassensível e do peptídeo natriurético cerebral sérico, a ressonância magnética cardíaca e o ecocardiograma com avaliação da deformação miocárdica (ecocardiograma strain) ${ }^{4}$.

Apesar da maior sensibilidade de detecçáo da injúria cardíaca nos métodos clássicos, os métodos contemporâneos apresentam fragilidades e limitaçóes que impedem o consenso da sua utilização na prática clínica ${ }^{4}$. A falta de estudos clínicos, que demonstrem o real benefício de adotar avaliaçóes cardíacas mais dispendiosas frente à decisão de iniciar ou interromper uma terapia oncológica, parece ter papel importante na divisão de opinióes 5 .
Neste artigo, serão discutidos os métodos clássicos e contemporâneos no diagnóstico, rastreio e manejo da cardiotoxicidade, relacionados ao tratamento oncológico.

\section{CARDIOTOXICIDADE}

A incidência geral da cardiotoxicidade é influenciada por uma combinação entre os fatores do indivíduo (comorbidades) e as características do tratamento oncológico (agentes, esquema de administração, área incluída no campo de radioterapia) ${ }^{6}$.

As terapias antineoplásicas podem elevar ao risco de desenvolvimento de insuficiência cardíaca congestiva em 15 vezes; ao de doenças cardiovasculares em dez vezes; e ao de acidentes vasculares encefálicos em nove vezes, com incremento na mortalidade tardia, conforme descrito por Armstrong et al. ${ }^{7}$, que avaliaram uma população infantojuvenil após 15-25 anos do tratamento oncológico, revelando um risco 8,2 vezes maior de morte cardíaca quando comparada a uma populaçáo pareada por sexo, náo exposta e da mesma faixa etária, além de morbidade considerável ${ }^{7}$.

De uma maneira mais ampla, a disfunção cardíaca pode ser classificada como aguda, subaguda e crônica quando baseada no tipo de alteração histológica e na evolução clínica relacionada aos tratamentos oncológicos em tipos I e II (Quadro 1), com impacto em modificaçáo de conduta frente à terapia antineoplásica ${ }^{8}$.

As manifestaçôes clínicas mais graves relacionadas à cardiotoxicidade são a disfunçáo e a insuficiência cardíacas.

\footnotetext{
1 Oncologista Clínico e Mestre em Oncologia Molecular. Cambridge University. Early Phase Trials Unit. Cambridge (UK), Inglaterra. Orcid iD: https://orcid.org/0000-0001-6427-229X

${ }^{2}$ Escola de Medicina da Universidade de Vassouras. Vassouras (RJ), Brasil. Orcid iD: https://orcid.org/0000-0002-7305-3504

${ }^{3}$ Escola de Medicina da Universidade de Vassouras. Vassouras (RJ), Brasil. Orcid iD: https://orcid.org/0000-0002-6832-9668

${ }^{4}$ Escola de Medicina da Universidade de Vassouras. Vassouras (RJ), Brasil. Orcid iD: https://orcid.org/0000-0002-7190-9196

${ }^{5}$ Escola de Medicina da Universidade de Vassouras. Vassouras (RJ), Brasil. Orcid iD: https://orcid.org/0000-0002-8013-7191

${ }^{6}$ Escola de Medicina da Universidade de Vassouras. Vassouras (RJ), Brasil. Orcid iD: https://orcid.org/0000-0003-2412-4182

${ }^{7}$ Escola de Medicina da Universidade Estácio de Sá. Rio de Janeiro (RJ), Brasil. Orcid iD: https://orcid.org/0000-0002-1233-4185

${ }^{8}$ Unidade de Pesquisa Clínica em Câncer de Mama do Hospital do Câncer III do Instituto Nacional de Câncer José Alencar Gomes da Silva (HCIII/INCA). Rio de Janeiro (RJ), Brasil. Orcid iD: https://orcid.org/0000-0002-5463-5517

Endereço para correspondência: Bruno de Henrique Rala de Paula. Early Phase Trials Unit. 11, Lingholme Close, Cambridge (UK) - CB4 3HW.E-mail: brunobhrp@hotmail.com
} 
Raramente, isquemia miocárdica, arritmias ventriculares e supraventriculares, hipertensão arterial, pericardite e eventos tromboembólicos podem evoluir de forma desfavorável $^{9}$ (Tabela 1$)$.

Quadro 1. Classificação para cardiomiopatia associada ao uso de quimioterápicos

\begin{tabular}{|c|c|c|c|c|}
\hline Cardiotoxicidade & Agente oncológico & $\begin{array}{c}\text { Relação com dose } \\
\text { cumulativa }\end{array}$ & $\begin{array}{c}\text { Achados na biópsia } \\
\text { endomiocárdica }\end{array}$ & $\begin{array}{c}\text { Reversibilidade } \\
\text { da injúria }\end{array}$ \\
\hline Tipo I & $\begin{array}{c}\text { Antraciclinas } \\
\text { Alquilantes }\end{array}$ & Sim & $\begin{array}{c}\text { Vacúolos, destruição } \\
\text { dos sarcômeros }\end{array}$ & Não \\
\hline Tipo II & $\begin{array}{c}\text { Anticorpos monoclonais } \\
\text { Inibidores da tirosina } \\
\text { quinase }\end{array}$ & Não & $\begin{array}{c}\text { Sem aparente } \\
\text { destruição celular }\end{array}$ & $\begin{array}{c}\text { Sim (maioria dos } \\
\text { casos) }\end{array}$ \\
\hline
\end{tabular}

Tabela 1. Toxicidade cardiovascular dos principais agentes antineoplásicos utilizados

\begin{tabular}{|c|c|c|c|}
\hline Agentes antineoplásicos & Efeitos cardiovasculares & Principais fisiopatologia & Incidência \\
\hline $\begin{array}{l}\text { Antraciclinas }^{10} \text { (doxorrubicina, } \\
\text { epirrubicina, Idarrubicina) }\end{array}$ & $\begin{array}{l}\text { Disfunção ventricular } \\
\text { esquerda e direita }\end{array}$ & $\begin{array}{c}\text { Peroxidação lipídica, stress } \\
\text { oxidativo }\end{array}$ & $7 \%-26 \%$ \\
\hline $\begin{array}{l}\text { Fluorpirimidinas }{ }^{11} \text { (capecitabina, } \\
\text { 5-fluourouracil) }\end{array}$ & $\begin{array}{l}\text { Cardiomiopatia isquêmica e } \\
\text { arritmias ventriculares }\end{array}$ & $\begin{array}{l}\text { Disfunção endotelial e vaso } \\
\text { espasmo coronariano }\end{array}$ & $1 \%-19 \%$ \\
\hline $\begin{array}{l}\text { Alquilantes }{ }^{12} \text { (ciclofosfamida em } \\
\text { altas doses, ifosfamida, platinas) }\end{array}$ & $\begin{array}{l}\text { Miocardite hemorrágica, } \\
\text { pericardite aguda letal }\end{array}$ & $\begin{array}{c}\text { Lesão cardíaca oxidativa } \\
\text { direta }\end{array}$ & $7 \%-28 \%$ \\
\hline Taxanes $^{13}$ (paclitaxel, docetaxel) & $\begin{array}{l}\text { Bradicardia sinusal, } \\
\text { fibrilação atrial, arritmias } \\
\text { ventriculares e isquemia } \\
\text { miocárdica }\end{array}$ & $\begin{array}{l}\text { Polimerização de tubulinas } \\
\text { levando à disfunção } \\
\text { de microtúbulos com } \\
\text { distúrbios na divisão celular } \\
\text { e liberação maciça de } \\
\text { histamina }\end{array}$ & $<0,1 \%-31 \%$ \\
\hline $\begin{array}{l}\text { Bloqueador do receptor de HER-2 }{ }^{14} \\
\text { (trastuzumabe) }\end{array}$ & $\begin{array}{l}\text { Insuficiência ventricular } \\
\text { esquerda }\end{array}$ & $\begin{array}{l}\text { Alteração da integridade } \\
\text { mitocondrial levando à } \\
\text { disfunção da contratilidade } \\
\text { sem alterações profundas } \\
\text { na ultraestrutura dos } \\
\text { cardiomiócitos }\end{array}$ & $1 \%-27 \%$ \\
\hline $\begin{array}{l}\text { Inibidor da angiogênese }{ }^{15} \\
\text { (bevacizumabe) }\end{array}$ & $\begin{array}{l}\text { Hipertensão arterial } \\
\text { e fenômenos } \\
\text { tromboembólicos }\end{array}$ & $\begin{array}{c}\text { Etiologia adrenérgica ou } \\
\text { renovascular. Aumento } \\
\text { da disfunção das células } \\
\text { endoteliais e redução } \\
\text { do óxido nítrico e de } \\
\text { prostaglandinas }\end{array}$ & $1,7 \%-3,0 \%$ \\
\hline $\begin{array}{l}\text { Inibidores de tirosina quinase }{ }^{16} \\
\text { (sorafenibe, sunitinibe) }\end{array}$ & $\begin{array}{l}\text { Hipertensão arterial, } \\
\text { isquemia miocárdica, } \\
\text { insuficiência cardíaca e } \\
\text { disfunção miocárdica }\end{array}$ & $\begin{array}{l}\text { A hipertensão arterial se } \\
\text { relaciona com a inibição } \\
\text { do receptor do fator de } \\
\text { crescimento endotelial } \\
\text { vascular }\end{array}$ & $8 \%-28 \%$ \\
\hline $\begin{array}{l}\text { Inibidores imune de checkpoint }{ }^{17} \\
\text { (nivolumabe, pembrolizumabe, } \\
\text { atezolizumabe, avelumabe, } \\
\text { durvalumabe) }\end{array}$ & Pericardite e miocardite & $\begin{array}{l}\text { Aumento da resposta do } \\
\text { sistema imune }\end{array}$ & $1 \%-2 \%$ \\
\hline $\begin{array}{l}\text { Inibidor de quinase dependente } \\
\text { de ciclinas } 4 \text { e } 6^{18} \text { (ribociclibe) }\end{array}$ & $\begin{array}{c}\text { Bradicardia e } \\
\text { prolongamento do intervalo } \\
\text { QTc }\end{array}$ & $*$ & $<2 \%$ \\
\hline
\end{tabular}

Nota: ${ }^{*}$ Ainda sem dados suficientes. 
As novas drogas para o tratamento oncológico merecem atenção diferenciada, principalmente pelo padrão distinto de injúria. Os inibidores de checkpoint podem causar um espectro de toxicidades autoimunes como a miocardite e a pericardite ${ }^{17}$. Já os inibidores de quinases dependentes de ciclinas (CDK) 4 e 6 , podem induzir arritmias cardíacas por prolongar o intervalo $\mathrm{QTc}^{18}$. Nestes dois últimos grupos de drogas, a incidência parece ser baixa, porém estudos encontram-se em andamento para entendimento do mecanismo causal, qual rastreamento e manejo ideal da cardiotoxicidade, seja preventivo e ou terapêutico.

\section{MÉTODOS CLÁSSICOS}

A avaliação inicial do risco de desenvolvimento de complicaçóes cardíacas antes de administrar a terapia oncológica é crucial $^{19}$.

$\mathrm{Na}$ anamnese, procuram-se fatores de risco para o desenvolvimento de disfunçôes cardíacas como diabetes, doença renal, doença valvar, hipertensão arterial, insuficiência cardíaca e/ou arritmias prévias ${ }^{20}$.

A classificação do New York Heart Association (NYHA) de 1994 serve de parâmetro comparativo durante o tratamento, sendo uma forma indireta de avaliar clinicamente o nível de disfunçâo cardíaca desenvolvida ${ }^{21}$. É incomum pacientes evoluírem para NYHA III ou IV durante o tratamento oncológico, e a diminuição da função do ventrículo esquerdo pode ser avaliada na fase assintomática por meio do ecocardiograma ${ }^{22}$.

O eletrocardiograma é útil na avaliação de arritmias e eventos vasculares prévios, porém limitado na avaliação da função cardiovascular. Apesar de mundialmente disponível, é frequentemente subutilizado. É importante para monitorização de alteraçôes prévias, pois o surgimento de novas alterações pode sugerir injúria cardiovascular aguda e/ou subaguda ${ }^{23}$. As principais variações incluem alteração da repolarização ventricular, intervalo QT, síndromes coronarianas agudas, arritmias supraventriculares e ventriculares, sendo ainda possível sugerir pericardite e miocardite ${ }^{24}$.

Atualmente, consideram-se a função sistólica e a FEVE como avaliaçóes cruciais no rastreio ótimo da cardiotoxicidade ${ }^{4}$. Podem ser medidas tanto pelo ecocardiograma bidimensional quanto pelo MUGA, exames de imagem realizados rotineiramente na prática clínica $^{25}$.

Os dois métodos têm suas limitaçôes. Enquanto a sensibilidade de detecção das disfunçóes ventriculares pode estar diminuída no ecocardiograma bidimensional em pacientes com obesidade e doença pulmonar, no MUGA pode haver falha na mensuração da FEVE em pacientes com arritmias ${ }^{26}$. Adicionalmente, o ecocardiograma transtorácico apresenta relação custo-benefício favorável e inócua ao paciente, sendo eventualmente utilizado inclusive como método alternativo na confirmaçáo de algumas mensuraçôes em que o MUGA é limitado e requer exposição à radiação ${ }^{27}$.

\section{MÉTODOS CONTEMPORÂNEOS}

\section{TROPONINA ULTRASSENSÍVEL}

É um biomarcador composto de diversas subunidades cuja elevação é altamente sensível para lesão miocárdica, sem, entretanto, identificar a causa clínica da injúria celular $^{28}$. Witteles ${ }^{29}$, para avaliar a validade da troponina I como biomarcador de detecçáo precoce de toxicidade cardíaca a várias drogas antineoplásicas, concluiu em sua revisão que a troponina I pode servir como um marcador de suscetibilidade à toxicidade cardíaca somente para alguns tipos de pacientes ${ }^{29}$. Resultados positivos também foram documentados com a troponina T, em que o aumento da concentração sérica logo após a administração de alguns quimioterápicos associou-se ao risco subsequente de anormalidades do ventrículo esquerdo, como, por exemplo, redução na espessura da parede e dilatação. Além disso, os níveis séricos de troponina $\mathrm{T}$ aumentam conforme a dose acumulada e a gravidade da injúria.

Apesar de não empregada rotineiramente, a dosagem da troponina plasmática pode ser utilizada como marcador precoce de cardiotoxicidade e espera-se que futuramente possa orientar modificaçóes nos regimes terapêuticos ${ }^{30}$.

\section{Peptídeo natriurético Cerebral}

O fator natriurético atrial, o peptídeo natriurético cerebral, e o peptídeo natriurético tipo $\mathrm{C}$ constituem a família dos peptídeos natriuréticos, que têm papel importante na homeostase cardiovascular e na modulação do crescimento celular. As concentraçôes plasmáticas do fator natriurético atrial e do peptídeo natriurético tipo-B aumentam em resposta à distensão do tecido atrial e parecem ser antagonistas dos efeitos da angiotensina II no tônus vascular, frequentemente envolvidos na fisiopatologia das cardiopatias ${ }^{31}$.

Dores et al. ${ }^{32}$ conduziram um estudo prospectivo para a detecção precoce de cardiotoxicidade induzida pelo trastuzumabe, utilizando a concentração plasmática do peptídeo natriurético tipo B e da FEVE. Não houve diferença significativa entre a FEVE pré-tratamento e três meses após, bem como na concentração do peptídeo natriurético tipo B; mas, em razão da pequena amostra, novos estudos são necessários para uma conclusáo definitiva ${ }^{32}$. 


\section{ECOCARDIOGRAMA COM AVALIAÇÃO DA DEFORMIDADE MIOCÁRDICA (STRAIN)}

O rastreamento speckle tracking, agora disponível em vários sistemas ecocardiográficos, permite avaliar os diferentes componentes da deformação miocárdica - strain longitudinal, radial e circunferencial ${ }^{33}$.

O strain longitudinal global é sensível para detectar alterações precoces da função ventricular antes de manifestaçôes clínicas e da alteração da FEVE. No entanto, ainda não há uma padronização de seu uso e do ponto de corte a ser utilizado como preditor de cardiotoxicidade ${ }^{34}$.

\section{RESSONÂNCIA NUCLEAR MAGNÉTICA CARDÍACA}

Método considerado padrão-ouro para a avaliação dos volumes, da massa e da FEVE, sendo o procedimento de escolha para detecçáo de inflamação, necrose e fibrose miocárdica com alta resolução ${ }^{35,36}$.

Tem sensibilidade elevada para detectar deterioração precoce da função cardíaca e alteraçóes miocárdicas, mesmo subclínicas. Além de altamente reprodutível, permite a avaliação funcional e perfusional miocárdica, sendo útil inclusive em pacientes com janela ecocardiográfica limitada ${ }^{37}$.

Entretanto, o custo elevado, a necessidade de exames repetidos e a limitada disponibilidade dificultam o seguimento clínico de rotina. Adicionalmente, não há consenso sobre qual conduta a ser adotada quando se identificam alteraçôes subclínicas diagnosticadas em pacientes oncológicos ${ }^{38}$.

\section{CENÁRIO ATUAL E PERSPECTIVAS FUTURAS}

Apesar de consolidada, a prática clínica ainda carece de recomendaçôes de rastreio da cardiotoxicidade baseadas em evidência. Tanto para os métodos complementares quanto para avaliação clínica, as diretrizes vigentes somente mimetizam os regimes de rastreio utilizados nos estudos pivotais.

O possível prejuízo, gerado com interrupçóes temporárias de esquemas terapêuticos oncológicos, potencialmente curativos, baseando-se em um resultado de exame complementar suspeito de injúria cardíaca em pacientes assintomáticos, deve ser levado em consideração, como o observado em pacientes portadoras de câncer de mama em tratamento adjuvante com trastuzumab ${ }^{39,40}$.

Com o advento dos métodos contemporâneos, ganhou-se em sensibilidade de detecção de alteraçóes cardíacas incipientes, mas ainda não se pode utilizar esses resultados para modificação da conduta oncológica, diferentemente dos métodos radiológicos convencionais ${ }^{5}$. Vale ressaltar que a agregação dessas novas tecnologias contribui de forma crucial para a distinção entre cardiotoxicidade relacionada aos antineoplásicos ou a outras causas, que eventualmente podem ser curáveis e/ ou reversíveis ${ }^{30}$.

Portanto, o rastreamento da cardiotoxicidade continua baseado na abordagem clássica. Os métodos contemporâneos parecem ter indicação de exceção na prática clínica, requerendo individualização do paciente por uma equipe multidisciplinar ${ }^{41}$.

Estudos publicados e outros em andamento são destinados à esfera da prevençáo baseada no uso de medicaçôes que, porventura, reduziriam o dano cardíaco (inibidores da enzima conversora de angiotensina, betabloqueadores $)^{42}$. Outras linhas de pesquisa exploram o melhor manejo de pacientes com doença miocárdica preexistente $^{43}$.

Evidências sobre a sensibilidade, especificidade e análise de custo em se adotar os métodos clássicos combinados ou não aos métodos contemporâneos de rastreio são aguardadas, para assim fornecer dados ligados à prevenção, à monitorização e à tomada de conduta dos pacientes oncológicos que estáo prologando a sobrevida, muito em parte em razão das novas drogas e das mudanças nos hábitos de vida.

\section{CONCLUSÃO}

Os métodos contemporâneos apresentam maior sensibilidade na detecçáo de cardiotoxicidade do que os clássicos. O custo elevado, o acesso difícil e o pouco consenso em abordagem das alteraçôes subclínicas são principais fatores que limitam sua agregação na prática clínica. Estudos prospectivos são aguardados para definição do regime de rastreio levando-se em consideração o acesso, o custo e a aplicabilidade clínica.

\section{CONTRIBUIÇÕES}

Os autores participaram de todas as etapas do manuscrito e da aprovação da versão final para publicação.

\section{DECLARAÇÃO DE CONFLITO DE INTERESSES}

Nada a declarar.

\section{FONTES DE FINANCIAMENTO}

Não há.

\section{REFERÊNCIAS}

1. Nurgali K, Thomas Jagoe R, Abalo R. Editorial: adverse effects of cancer chemotherapy: Anything new to improve tolerance and reduce sequelae? Front 
Pharmacol. 2018;9:245. doi: https://doi.org/10.3389/ fphar.2018.00245

2. Bhatt PK, Okwuosa T. The emergence of a new specialty of oncocardiology. Postgrad Med J. 2017;93(1096):59-60. doi: http://dx.doi.org/10.1136/ postgradmedj-2016-134440

3. Curigliano G, Cardinale D, Dent $S$, et al. Cardiotoxicity of anticancer treatments: epidemiology, detection, and management. CA Cancer J Clin. 2016;66(4):309-25. doi: https://doi.org/10.3322/caac.21341

4. Pondé NF, Lambertine M, Azambuja E. Twenty years of anti-HER2 therapy-associated cardiotoxicity. ESMO Open. 2016;1(4):e000073. doi: https://doi.org/10.1136/ esmoopen-2016-000073

5. Dang CT, Yu AF, Jones LW, et al. Cardiac surveillance guidelines for trastuzumab-containing therapy in earlystage breast cancer: getting to the heart of the matter. J Clin Oncol. 2016;34(10):1030-1033. doi: https://doi. org/10.1200/JCO.2015.64.5515

6. Aleman BM, Moser EC, Nuver J, et al. Cardiovascular disease after cancer therapy. EJC Suppl. 2014;12(1):1828. doi: https://doi.org/10.1016/j.ejcsup.2014.03.002

7. Armstrong GT, Kawashima T, Leisenring W, et al. Aging and risk of severe, disabling, life-threatening, and fatal events in the childhood cancer survivor study. J Clin Oncol. 2014;32(12):1218-27. doi: https://doi. org/10.1200/JCO.2013.51.1055

8. Henning RJ, Harbison RD. Cardio-oncology: cardiovascular complications of cancer therapy. Future Cardiol. 2017;13(4):379-396. doi: https://doi. org/10.2217/fca-2016-0081

9. Varma A. Cardio-oncology: an integrated discipline or just a portmanteau? J Lancaster General Health. 2017;12(4):100-111.

10. Nebigil CG, Désaubry L. Updates in anthracyclinemediated cardiotoxicity. Front Pharmacol. 2018;9:1262. doi: https://doi.org/10.3389/fphar.2018.01262

11. Kanduri J, More LA, Godishala A, et al. Fluoropyrimidineassociated cardiotoxicity. Cardiol Clin. 2019;37(4):399405. doi: https://doi.org/10.1016/j.ccl.2019.07.004

12. Gottdiener JS, Appelbaum FR, Ferrans VJ, et al. Cardiotoxicity associated with high-dose cyclophosphamide therapy. Arch Intern Med. 1981;141(6):758-763. doi: https://doi.org/10.1001/ archinte.1981.00340060066015

13. Madeddu C, Deidda M, Piras A, et al. Pathophysiology of cardiotoxicity induced by nonanthracycline chemotherapy. J Cardiovasc Med. 2016;17(Suppl 1):e12-e18. doi: https://doi.org/10.2459/JCM.0000000000000376

14. Mohan N, Jiang J, Dokmanovic M, et al. Trastuzumabmediated cardiotoxicity: current understanding, challenges, and frontiers. Antib Ther. 2018;1(1):13-17. doi: https://doi.org/10.1093/abt/tby003
15. Vaklavas C, Lenihan D, Kurzrock R, et al. Anti-vascular endothelial growth factor therapies and cardiovascular toxicity: what are the important clinical markers to target? Oncologist. 2010;15(2):130-141. doi: https:// doi.org/10.1634/theoncologist.2009-0252

16. Lynch KE, Lynch JA, Efimova O, et al. Cardiotoxicity of tyrosine kinase inhibitors among veterans diagnosed with renal cell carcinoma. J Clin Oncol. 2017;35(15 Suppl):e18248. doi: https://doi.org/10.1200/ JCO.2017.35.15_suppl.e18248

17. Lyon AR, Yousaf N, Battisti NML, et al. Immune checkpoint inhibitors and cardiovascular toxicity. Lancet Oncol. 2018;19(9):e447-e458. doi: https://doi. org/10.1016/S1470-2045(18)30457-1

18. Thill M, Schmidt M. Management of adverse events during cyclin-dependent kinase 4/6 (CDK4/6) inhibitorbased treatment in breast cancer. Ther Adv Med Oncol. 2018;10: 1758835918793326. doi: https://doi. org/10.1177/1758835918793326

19. Finet JE, Tang WHW. Protecting the heart in cancer therapy [version 1; peer review: 2 approved]. F1000Res. 2018;7(F1000FacultyRev):1566. doi: https://doi. org/10.12688/f1000research.15190.1

20. Schoormans D, Pedersen SS, Dalton S, et al. Cardiovascular co-morbidity in cancer patients: the role of psychological distress. Cardio-Oncology. 2016;2:9. doi: https://doi.org/10.1186/s40959-016-0019-x

21. New York Heart Association, Criteria Committee. Nomenclature and criteria for diagnosis of diseases of the heart and great vessels. 9th ed. Boston: Little, Brown; 1994.

22. Hahn VS, Lenihan DJ, Ky B. Cancer therapyinduced cardiotoxicity: basic mechanisms and potential cardioprotective therapies. J Am Heart Assoc. 2014;3(2):e000665. doi: https://doi.org/10.1161/ JAHA.113.000665

23. Pezo RC, Yan AT, Earle C, et al. Underuse of ECG monitoring in oncology patients receiving QT-interval prolonging drugs. Heart. 2019;105(21):1649-1655. doi: http://dx.doi.org/10.1136/heartjnl-2018-314674

24. Kleiman R, Litwin J, Morganroth J. Benefits of centralized ECG reading in clinical oncology studies. Ther Innov Regul Sci. 2016;50(1):123-129. doi: https:// doi.org/10.1177/2168479015597729

25. Yang SN, Sun SS, Zhang G, et al. Left ventricular ejection fraction estimation using mutual information on technetium-99m multiple-gated SPECT scans. Biomed Eng Online. 2015;14:119. doi: https://doi.org/10.1186/ s12938-015-0117-2

26. Pellikka PP, She L, Holly TA, et al. Variability in ejection fraction measured by echocardiography, gated singlephoton emission computed tomography, and cardiac magnetic resonance in patients with coronary artery disease and left ventricular dysfunction. JAMA Netw 
Open. 2018;1(4):e181456. doi: https://doi.org/10.1001/ jamanetworkopen.2018.1456

27. Wang CL, Chu PH. Echocardiography for Evaluation of Oncology Therapy-Related Cardiotoxicity. Acta Cardiol Sin. 2016;32(5):560-564. doi: https://doi.org/10.6515/ ACS20151024A

28. Johnston JR, Chase PB, Pinto JR. Troponin through the looking-glass: emerging roles beyond regulation of striated muscle contraction. Oncotarget. 2017;9(1):1461-1482. doi: https://doi.org/10.18632/oncotarget.22879

29. Witteles RM. Biomarkers as Predictors of Cardiac Toxicity From Targeted Cancer Therapies. J Card Fail. 2016;22(6):459-64. doi: https://doi.org/10.1016/j. cardfail.2016.03.016

30. Tan LL, Lyon AR. Role of biomarkers in prediction of cardiotoxicity during cancer treatment. Curr Treat Options Cardiovasc Med. 2018;20(7):55. doi: https:// doi.org/10.1007/s11936-018-0641-z

31. Buglioni A, Burnett JC Jr. Pathophysiology and the cardiorenal connection in heart failure. Circulating hormones: biomarkers or mediators. Clin Chim Acta. 2015;443(30):3-8. doi: https://doi.org/10.1016/j. cca.2014.10.027

32. Dores H, Abecasis J, Correia MJ, et al. Detection of early sub-clinical trastuzumab-induced cardiotoxicity in breast cancer patients. Arq Bras Cardiol. 2013;100(4):328-32. doi: https://doi.org/10.5935/abc.20130050

33. Portugal G, Branco LM, Galrinho A, et al. Importância da deformação longitudinal na deteção da cardiotoxicidade induzida por quimioterapia e na identificação de padrôes específicos de afetação segmentar. Rev Port Cardiol. 2017;36(1):9-15. doi: https://doi.org/10.1016/j. repc.2016.06.009

34. Charbonnel C, Convers-Domart R, Rigaudeau S, et al. Assessment of global longitudinal strain at low-dose anthracycline-based chemotherapy, for the prediction of subsequent cardiotoxicity. Eur Heart J Cardiovasc Imaging. 2017;18(4):392-401. doi: https://doi. org/10.1093/ehjci/jew223

35. Tamene AM, Masri C, Konety SH. Cardiovascular MR imaging in cardio-oncology. Magn Reson Imaging Clin N Am. 2015;23(1):105-16. doi: https://doi. org/10.1016/j.mric.2014.09.007.

36. Thavendiranathan P, Wintersperger BJ, Flamm SD, et al. Cardiac MRI in the assessment of cardiac injury and toxicity from cancer chemotherapy: a systematic review. Circ Cardiovasc Imaging. 2013;6(6):1080-1091 doi: https:// doi.org/10.1161/CIRCIMAGING.113.000899

37. Jordan JH, Todd RM, Vasu S, et al. Cardiovascular magnetic resonance in the oncology patient. J Am Coll Cardiol Img. 2018;11(8):1150-1172. doi: https://doi. org/10.1016/j.jcmg.2018.06.004

38. Meyersohn NNM, Pursnani A, Neilan TG. Detection of cardiac toxicity due to cancer treatment: role of cardiac
MRI. Curr Treat Options Cardiovasc Med. 2015;17:38. doi: https://doi.org/10.1007/s11936-015-0396-8

39. De Paula BHR, Costa MEF, Bines J. Trastuzumab treatment-related cardiotoxicity: a storm in a teacup. J Cancer Sci Ther. 2018;10. doi: https://doi. org/10.4172/1948-5956-C12-156

40. Kalil Filho R, Hajjar LA, Bacal F, et al. I Diretriz Brasileira de cardio-oncologia da Sociedade Brasileira de Cardiologia. Arq Bras Cardiol. 2011;96(2 Supl 1):1-52.

41. Han X, Zhou Y, Liu W. Precision cardio-oncology: understanding the cardiotoxicity of cancer therapy. NPJ Precis Oncol. 2017;1(1):31. doi: https://doi.org/10.1038/ s41698-017-0034-x

42. Zamorano JL, Lancellotti P, Rodriguez Muñoz D, et al. 2016 ESC Position Paper on cancer treatments and cardiovascular toxicity developed under the auspices of the ESC Committee for Practice Guidelines: the task force for cancer treatments and cardiovascular toxicity of the European Society of Cardiology (ESC). Eur Heart J. 2016;37(36):2768-2801. doi: https://doi.org/10.1093/ eurheartj/ehw211

43. Lynce F, Barac A, Geng X, et al. Prospective evaluation of the cardiac safety of HER2-targeted therapies in patients with HER2-positive breast cancer and compromised heart function: the SAFE-HEaRt study. Breast Cancer Res Treat. 2019;175(3):595-603. doi: https://doi.org/10.1007/s10549-019-05191-2

Recebido em 11/8/19 Aprovado em 12/11/19 